\title{
Highlights from the STAR experiment
}

\author{
Alexey Aparin ${ }^{1, *}$ for the STAR collaboration \\ ${ }^{1}$ Joint Institute for Nuclear Research, Dubna, Russia
}

\begin{abstract}
We present an overview of the recent results obtained by the STAR Collaboration at RHIC. We report the beam energy dependence of directed flow of strange particles. These results support the assumption, that directed flow is formed before hadronization and observed particles are formed via coalescence of constituent quarks. First evidence of a non-zero directed flow of $D^{0}$ meson is reported. We present the precision measurement of elliptic flow of $D^{0}$ meson and the first measurement of $\Lambda_{c}$ baryon in $\mathrm{Au}+\mathrm{Au}$ collisions at $\sqrt{s_{N N}}$ $=200 \mathrm{GeV}$, which suggests thermalization of charm quarks. We report results of global $\Lambda$ polarization measurements in $\mathrm{Au}+\mathrm{Au}$ collisions, together with the investigation of polarization dependence of centrality and transverse momentum $p_{T}$. Results from the STAR fixed-target program show good agreement with previously obtained results. A precise measurement of the ${ }_{\Lambda}^{3} H$ lifetime is presented. Mesonic decay modes are used to reconstruct the ${ }_{\Lambda}^{3} H$ from $\mathrm{Au}+\mathrm{Au}$ collisions. The measured lifetime is about $50 \%$ shorter than the one of a free $\Lambda$, indicating a strong hyperon-nucleon interaction in the hypernucleus system. Finally, we give an outlook to detector upgrades for the Beam Energy Scan phase II.
\end{abstract}

\section{Azimuthal anisotropy measurements}

One of the most important observables in heavy-ion experiments is the azimuthal anisotropic flow [1] that is usually quantified by the Fourier coefficients of the azimuthal distribution of the final state particles relative to the collision symmetry planes $E d^{3} N / d p^{3}=$ $1 / 2 \pi \cdot d^{2} N / p_{t} d p_{t} d \eta \cdot\left(1+\Sigma 2 v_{n} \cos \left[n\left(\phi-\Psi_{n}\right)\right]\right)$. The first-order coefficient, called the directed flow, is argued to be sensitive to the equation of state of the matter, and could serve as a possible signature of the QGP phase transition. STAR has recently performed directed flow measurements at mid-rapidity for $\Lambda, \bar{\Lambda}, K^{ \pm}, K_{s}^{0}$, and $\phi$ at $\sqrt{s_{N N}}=7.7,11.5,14.5,19.6$, 27, 39, 62.4 and $200 \mathrm{GeV}$ in $\mathrm{Au}+\mathrm{Au}$ collisions. Results show good sensitivity for testing a picture where the flow is assumed to be formed before hadron formation and the observed particles are assumed to form via coalescence of constituent quarks. Fig. 1 represents $v_{1}(y)$ for indicated particles at two centralities. One can see that within errors, the plotted species have a near linear $v_{1}(y)$ over the acceptance of the STAR detector.

Directed flow slope $d v_{1} / d y$ versus beam energy for $\Lambda, \bar{\Lambda}, K^{ \pm}, K_{s}^{0}, \phi$ was also studied [2] and compared with the data for $\pi^{ \pm}, p$ and $\bar{p}$ [3]. Combined data for the ten particle species available allow one to carry out a detailed investigation of constituent quark $v_{1}$. Obtained results support the assumption called the coalescence sum rule [4]. In this scenario the directed

\footnotetext{
*e-mail: aparin@jinr.ru
} 
flow $v_{1}$ is formed at the pre-hadronic stage, specific types of quarks have the same directed flow and the detected hadrons are formed via coalescence.

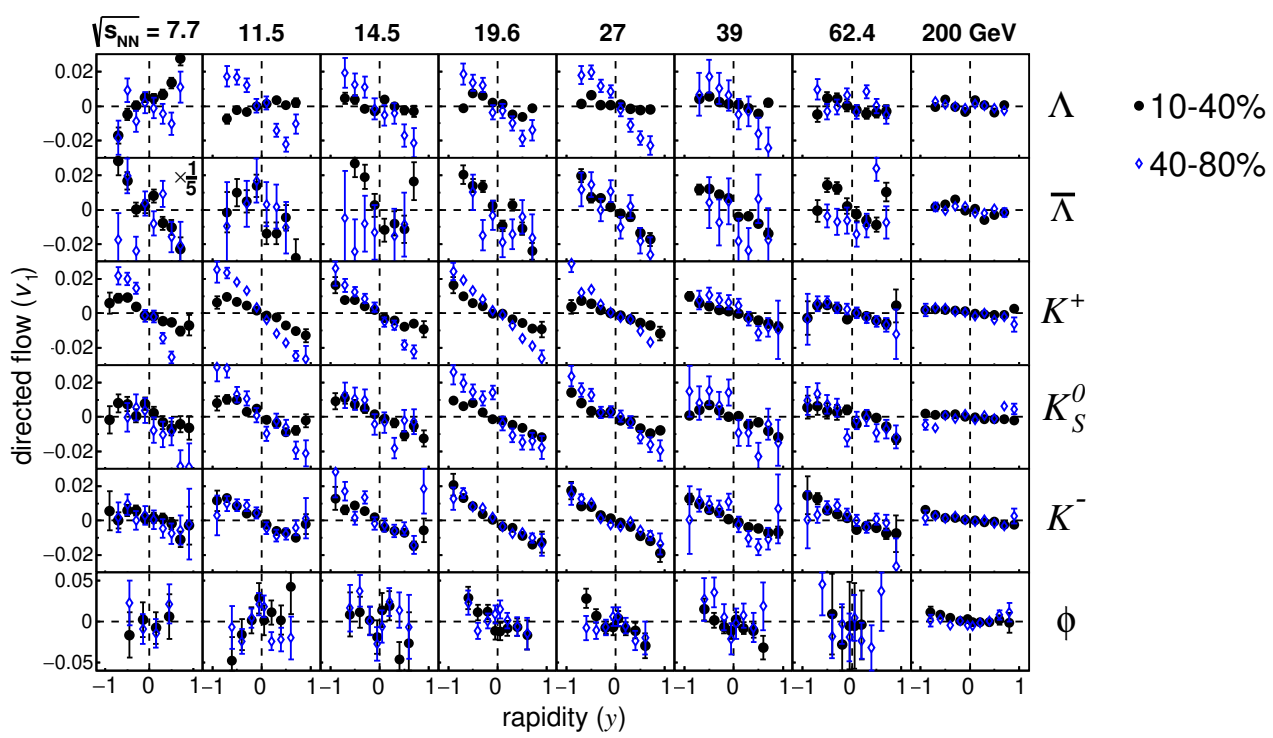

Figure 1. Directed flow as a function of rapidity for the six indicated particle species in $10-40 \%$ (black) and $40-80 \%$ (blue) central $\mathrm{Au}+\mathrm{Au}$ collisions at $\sqrt{s_{N N}}=7.7$ to $200 \mathrm{GeV}$. The error bars include statistical uncertainties only. All panels for species other than $\phi$ use the same $v_{1}$ scale with the exception of $\bar{\Lambda}$ at $\sqrt{s_{N N}}=7.7 \mathrm{GeV}$, where $v_{1}$ magnitudes are exceptionally large and require the measurements to be divided by five

Direct measurements of charmed hadrons require a very good vertex detector due to their very short lifetimes. STAR had installed the Heavy Flavor Tracker (HFT) [5], the MAPSbased vertex detector for run years 2014-2016. This allowed one to perform precise measurements of the flow for heavy flavor particles [6].

Fig. 2 shows the first measurement of the directed flow for charmed meson $D^{0}$ produced in $\mathrm{Au}-\mathrm{Au}$ collisions for $10-80 \%$ centrality. It is clear that within the error value of $v_{1}(y)$ is nonzero and follows the linear trend obtained previously for the light particle sector. The second harmonic of the charmed meson $D^{0}$ has been recently studied using data from run years 2014 and 2016. The measured $D^{0} v_{2}$ in $0-80 \%$ centrality $\mathrm{Au}+\mathrm{Au}$ collisions can be described by a viscous hydrodynamic calculation for transverse momentum $p_{T}$ less than $4 \mathrm{GeV} / \mathrm{c}$. The $D^{0}$ $v_{2}$ as a function of transverse kinetic energy $\left(m_{T}-m_{0}\right.$, where $\left.m_{T}=\sqrt{p_{T}^{2}+m^{2}}\right)$ is consistent with that of light mesons in $10-40 \%$ centrality $\mathrm{Au}+\mathrm{Au}$ collisions. These results suggest that charm quarks have achieved local thermal equilibrium with the medium produced in these collisions.

The energy dependent measurements reported here will be enhanced after STAR acquires greatly increased statistics using the upgraded detectors in Phase-II of the RHIC Beam Energy Scan (BES-II). 


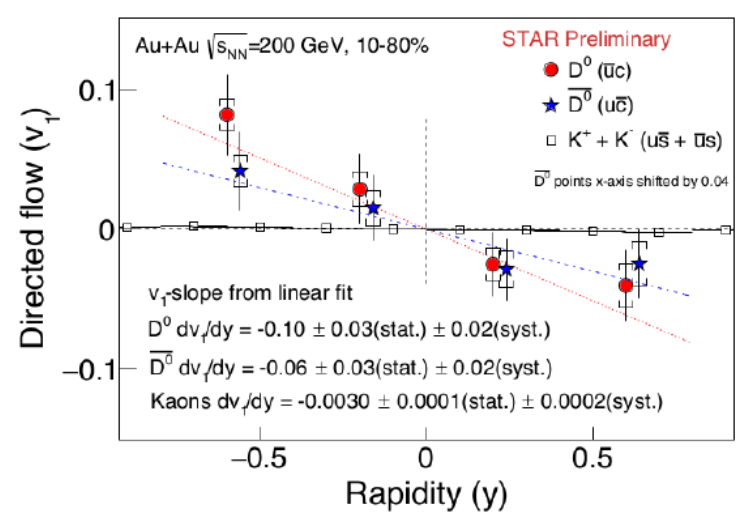

Figure 2. Directed flow as a function of rapidity for $D^{0}$ mesons in 10-80\% centrality $\mathrm{Au}+\mathrm{Au}$ collisions at $\sqrt{s_{N N}}=200 \mathrm{GeV}$ compared with directed flow of $K^{+}+K^{-}$. Data points for $\overline{D^{0}}$ are slightly shifted for visibility

\section{Heavy flavor production}

The excellent HFT resolution enabled a study of charmed hadrons with even smaller decay lengths than $D^{0}$, in particular $\Lambda_{c}$. Fig. 3 shows the reconstructed invariant mass of $p K \pi$ in $10-60 \% \mathrm{Au}+\mathrm{Au}$ collisions at $\sqrt{s_{N N}}=200 \mathrm{GeV}$, with topological cuts optimized for $\Lambda_{c}$. A peak at the $\Lambda_{c}$ mass is observed clearly. STAR has performed the first measurements of centrality dependence of $\Lambda_{c}$ production in heavy ion-collisions. It has been found that $\Lambda_{c} / D^{0}$ ratio increases from peripheral to central, that indicates hot medium effects. The measured STAR data point was compared to model calculations with various levels of charm quark thermalization in the medium and different coalescence implementations of the coalescence mechanism.

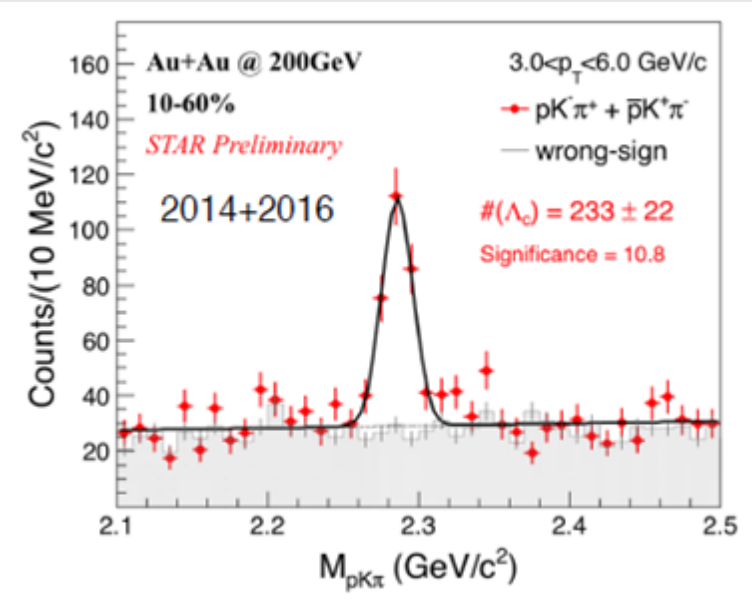

Figure 3. Reconstructed invariant mass of $p K \pi$ for $10-60 \%$ centrality $\mathrm{Au}+\mathrm{Au}$ collisions from run years 2014 and 2016, representing signal of $\Lambda_{c}$ baryons 
The energy loss of charm and bottom quarks is of great interest since quarks are expected to exhibit different radiative energy losses depending on their mass. The nuclear modification factor $\left.R_{A A}\left(p_{T}\right)=\sigma_{\text {in }}^{p p} /<N_{\text {coll }}^{A A}\right\rangle \cdot\left[d^{2} N_{A A} / d p_{T} d \eta\right] /\left[d^{2} \sigma_{p p} / d p_{T} d \eta\right]$ of inclusive $J / \psi$ via the di-muon channel at midrapidity in $0-40 \% \mathrm{Au}+\mathrm{Au}$ collisions at $\sqrt{s_{N N}}=200 \mathrm{GeV}$ was compared to LHC results [7]. The strong suppression at high $p_{T}$ obtained at RHIC indicates significant $J / \psi$ dissociation.

STAR has shown $\Upsilon R_{A A}$ for the $2 S+3 S$ states for $\mathrm{Au}+\mathrm{Au}$ collisions at $\sqrt{s_{N N}}=200 \mathrm{GeV}$ as a function of particles number $N_{\text {part }}$, compared to CMS data. There are indications that the suppression is weaker at $\sqrt{s_{N N}}=200 \mathrm{GeV}$ than at $\sqrt{s_{N N}}=2.76 \mathrm{TeV}$, which might be due to the lower temperature at RHIC. Weaker suppression of the $1 \mathrm{~S}$ state compared to the $2 \mathrm{~S}+3 \mathrm{~S}$ states in central collisions is consistent with the picture of sequential melting.

\section{Global polarization measurements}

STAR has recently reported measurements of global $\Lambda$ polarization in $\mathrm{Au}+\mathrm{Au}$ collisions $[8,9]$. The existence of alignment between the angular momentum of a non-central collision and the spin of emitted particles was demonstrated for the first time, revealing that the fluid produced in heavy-ion collisions is by far the most vortical system ever observed. The measured effect for $\Lambda$ and $\bar{\Lambda}$ hyperons has shown positive polarization of the order of a few percent for energies of BES and of tens of percent for the highest RHIC energy. The signal increases with decreasing collision energy, and systematic splitting between $\Lambda$ and $\bar{\Lambda}$ is observed which might indicate an additional magnetic component.

Spin-orbit coupling can generate a spin alignment, or polarization, along the direction of the vorticity which is on the average parallel to the overall angular momentum of the system. Fig. 4 shows the measured global polarization of $\Lambda$ and $\bar{\Lambda}$ as a function of the collision energy for the $20-50 \%$ centrality bin in Au+Au collisions. A set of different theoretical models is argued to describe the measured polarization [10]-[12].

Calculations for primary $\Lambda$ and all $\Lambda$ taking into account the effect of feed-down, from a $(3+1)$ D viscous hydrodynamic model vHLLE with the UrQMD initial state [10] are shown for comparison. The model calculations agree with the data over a wide range of collision energy, including $\sqrt{s_{N N}}=200 \mathrm{GeV}$ within the current accuracy of experimental measurements. Calculations from a Multi-Phase Transport (AMPT) model [11] predict slightly higher polarization than the hydrodynamic model, but are also in good agreement with the data within uncertainties.

Fig. 5 shows differential measurements of the polarization, versus the collision centrality, and hyperon's transverse momentum. With the given large uncertainties, it is not clear if the polarization saturates or even starts to drop off in the most peripheral collisions (Fig. 5, left). The polarization dependence on $p_{T}$ is weak or absent (Fig. 5, right), considering the large uncertainties, which is consistent with the expectation that the polarization is generated by a rotation of the system. No significant dependence on pseudorapidity or transverse momentum was observed. The statistical uncertainties need to be improved to reach a definitive conclusion on the event-by-event charge asymmetry, which is consistent with a possible contribution to the global polarization from the axial current induced by the initial magnetic field.

\section{Results from the fixed-target program}

STAR has demonstrated the capability to operate at very low $\sqrt{s_{N N}}=4.5 \mathrm{GeV}$ in the fixedtarget mode. A test run with Au-target installed $2 \mathrm{~m}$ from the TPC center within the beam 

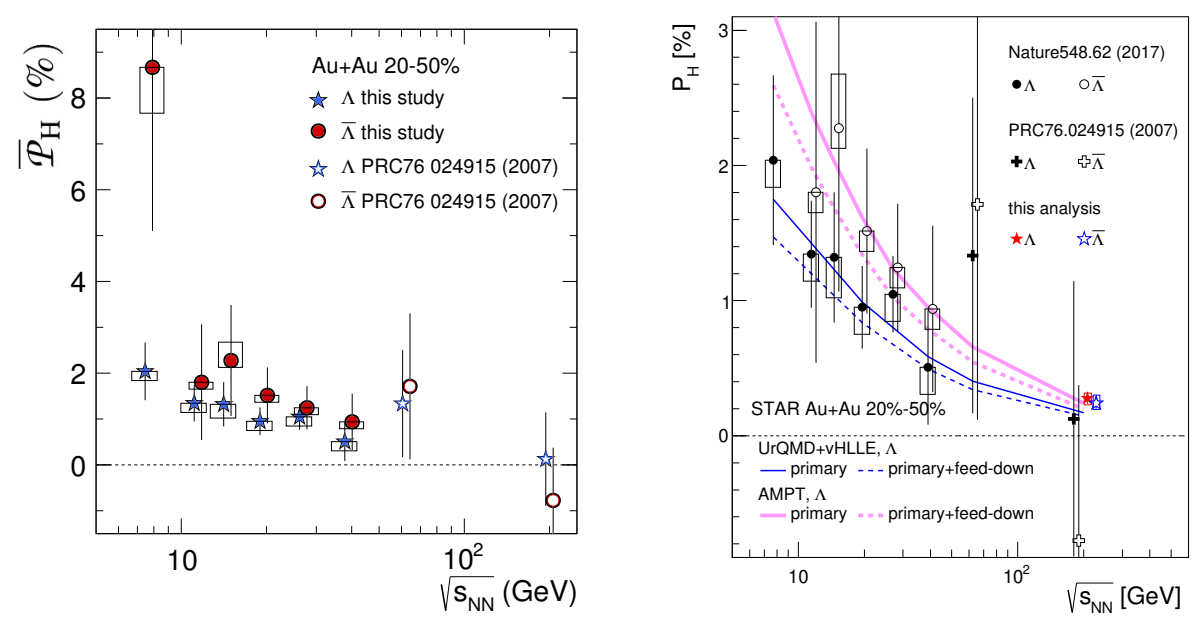

Figure 4. Global polarization $\bar{P}_{H}$ (where $\mathrm{H}=\Lambda$ or $\bar{\Lambda}$ ) of $\Lambda$ and $\bar{\Lambda}$ as a function of the collision energy $\sqrt{s_{N N}}$ for $20-50 \%$ centrality $\mathrm{Au}+\mathrm{Au}$ collisions. Left. The results of the measurements for BES energy range $\left(\sqrt{s_{N N}}<40 \mathrm{GeV}\right)$ are compared with the data previously calculated for 62.4 and $200 \mathrm{GeV}$ collisions, for which only statistical errors are plotted. Boxes indicate systematic uncertainties. Right. Thin lines show calculations from a (3+1)D cascade + viscous hydrodynamic model (UrQMD+vHLLE) [10] and bold lines show the AMPT model calculations [11]. In the case of each model, primary $\Lambda$ with and without the feed-down effect are indicated by dashed and solid lines, respectively. Open boxes and vertical lines show systematic and statistical uncertainties, respectively. Data points at $200 \mathrm{GeV}$ and for $\bar{\Lambda}$ are slightly horizontally shifted for visibility

pipe has been done recently. During 3 minutes run $1 \mathrm{M}$ events of $0-30 \%$ centrality $\mathrm{Au}+\mathrm{Au}$ were recorded. Fig. 6 shows energy dependence of the measured directed flow slope parameter $d v_{1} /\left.d y\right|_{y=0}$, comparing the data from the fixed-target mode to BES STAR [13] and AGS results [14]. Within the uncertainties the data are consistent with those measured in a collider experiments. Fig. 6, left, shows the slope parameter for the lightest mesons $\left(\pi^{ \pm}, K^{ \pm}\right.$and $\left.K_{S}^{0}\right)$ and Fig. 6, right, the same for lightest baryons $(p, \Lambda$ and $\bar{p})$.

Fig. 7 demonstrates STAR capabilities for particle identification in the fixed-target regime for strange particles. Spectra are plotted in dependence on transverse kinetic energy for $K_{S}^{0}$, left, and $\Lambda$, right.

\section{Hypertriton lifetime measurements}

STAR has performed a precise measurement of the ${ }_{\Lambda}^{3} H$ and ${ }_{\bar{\Lambda}}^{3} \bar{H}$ lifetime. Mesonic decay modes were used to reconstruct ${ }_{\Lambda}^{3} H$ from Au+Au collisions data. All ${ }_{\Lambda}^{3} H$ measurements, regardless of beam energy, were combined to increase the statistics. The hypertriton candidates were reconstructed from the invariant mass distributions of the daughters: ${ }^{3} \mathrm{He}+\pi^{-}$for the 2-body, and $d+p+\pi^{-}$for the 3-body decay channel of ${ }_{\Lambda}^{3} H$ and ${ }^{3} \bar{H} e+\pi^{+}$for the 2-body, and $\bar{d}+\bar{p}+\pi^{+}$for the 3 -body decay channel for ${ }_{\Lambda}^{3} H$ and ${ }_{\bar{\Lambda}}^{3} \bar{H}$, respectively.

A minimum $\chi^{2}$ estimation is used to determine the lifetime of $\tau=142+24-21$ (stat.) \pm 31 (syst.) ps. This lifetime is about 50\% shorter than the lifetime $\tau=263 \pm 2$ ps of a free $\Lambda$, indicating strong hyperon-nucleon interaction in the hypernucleus system. Fig. 8 shows the experimentally measured hypertriton lifetime. 

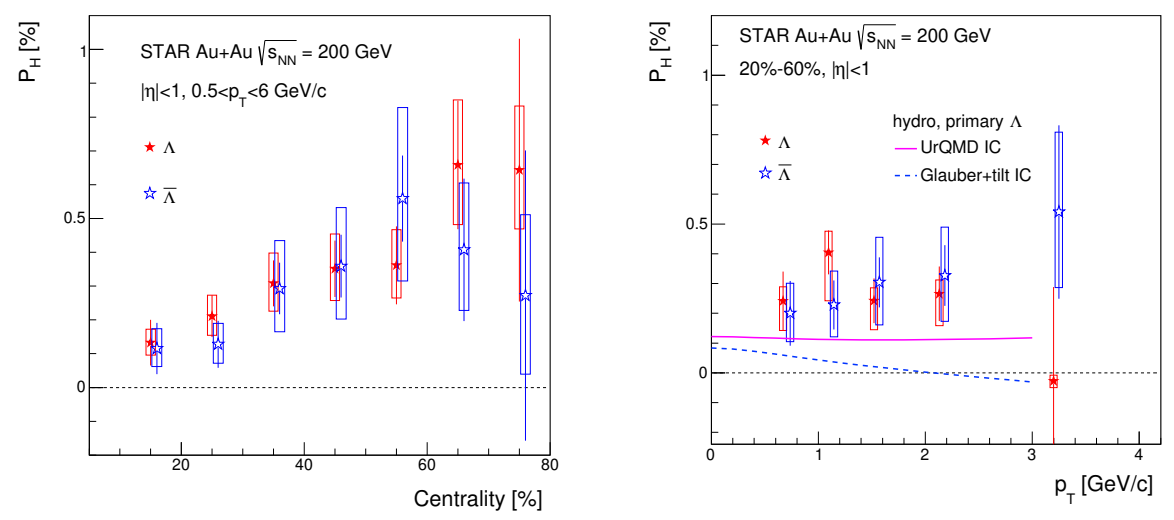

Figure 5. Left. Polarization of $\Lambda$ and $\bar{\Lambda}$ as a function of the collision centrality in $\mathrm{Au}+\mathrm{Au}$ collisions at $\sqrt{s_{N N}}=200 \mathrm{GeV}$. Right. Polarization of $\Lambda$ and $\bar{\Lambda}$ as a function of transverse momentum $p_{T}$ for the $20-60 \%$ centrality bin in $\mathrm{Au}+\mathrm{Au}$ collisions at $\sqrt{s_{N N}}=200 \mathrm{GeV}$. Hydrodynamic model calculations for $\Lambda$ with two different initial conditions are compared. Open boxes and vertical lines show systematic and statistical uncertainties. Data points for $\bar{\Lambda}$ are slightly shifted for visibility
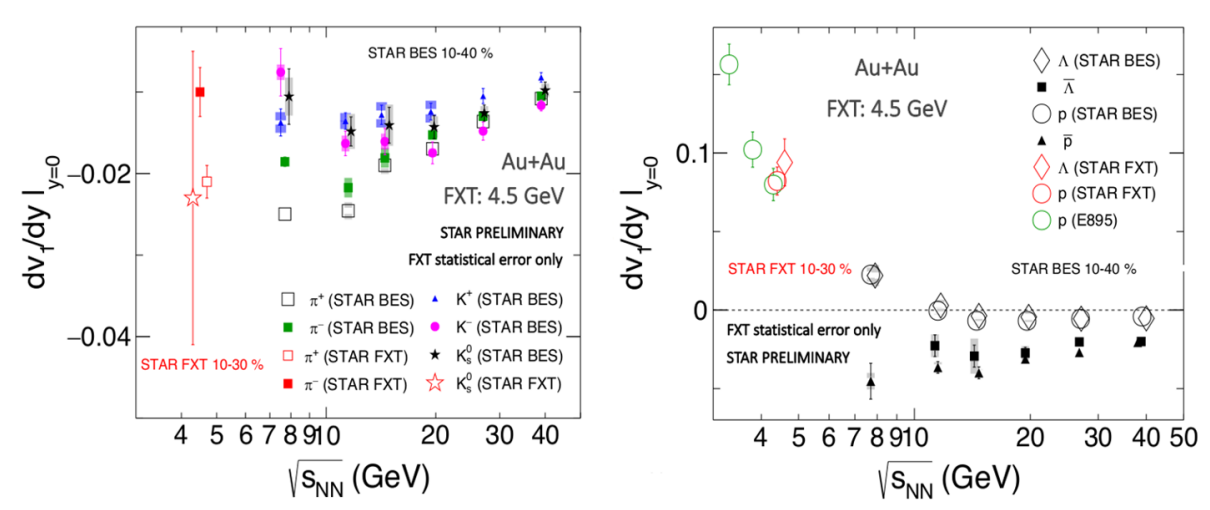

Figure 6. Directed flow slope at mid-rapidity as a function of the center-of-mass energy $\sqrt{s_{N N}}$ for the STAR fixed-target data. Left: $d v_{1} /\left.d y\right|_{y=0}$ for light mesons, compared to results obtained at STAR BES-I. Right: $d v_{1} /\left.d y\right|_{y=0}$ for light baryons, compared to results obtained at AGS and STAR BES-I

The STAR experiment will collect large datasets for $\mathrm{Au}+\mathrm{Au}$ collisions during BES phase II in years 2019-2020, which will further reduce experimental uncertainties on the ${ }_{\Lambda}^{3} H$ lifetime and will likely provide a new insight into its structure.

\section{Detector upgrades for BES-II}

BES phase II, planned to run in years 2019 and 2020, covers the energy range of $\sqrt{s_{N N}}<$ $20 \mathrm{GeV}$ in both the collider and fixed-target modes. The primary goals of BES-II are the 

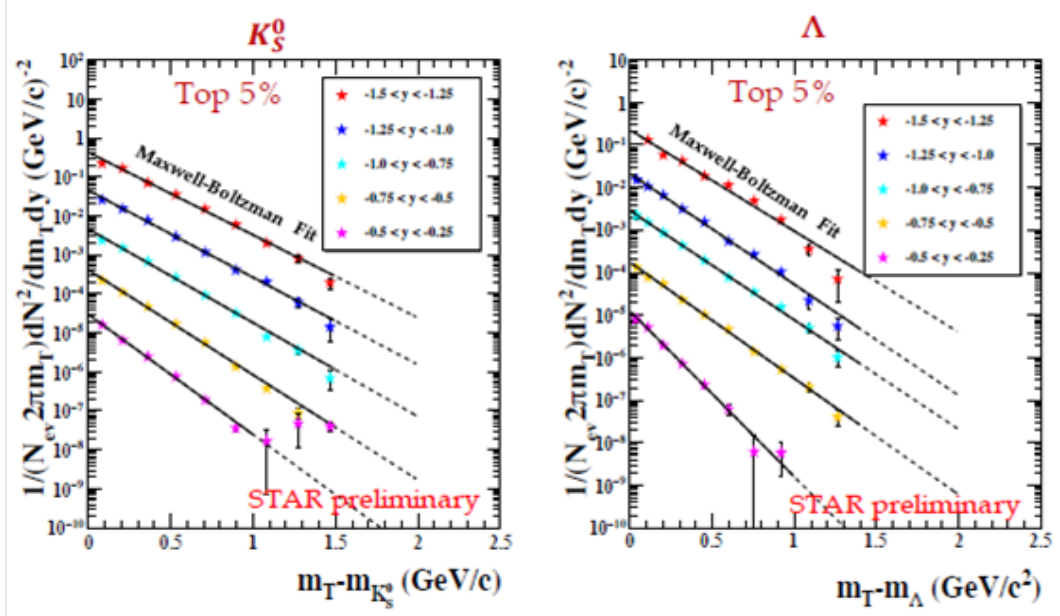

Figure 7. Spectra of strange particles obtained in the fixed-target mode as a function of transverse kinetic energy. Left: Spectra of $K_{S}^{0}$. Right: Spectra of $\Lambda$

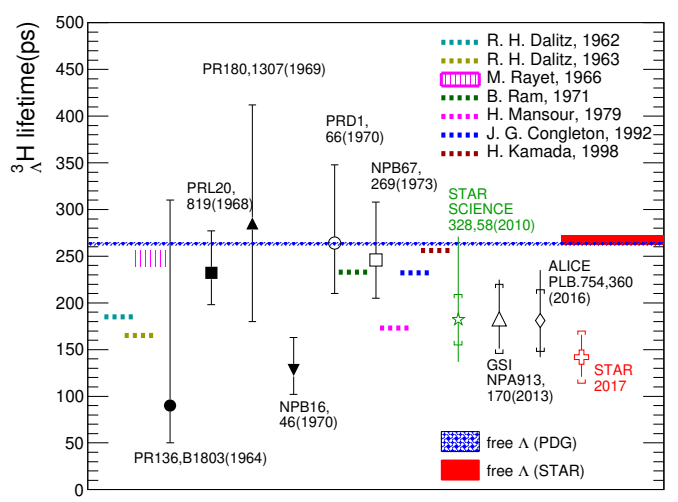

Figure 8. A summary of worldwide ${ }_{\Lambda}^{3} H$ lifetime experimental measurements and theoretical calculations. The two star markers are the STAR results published in 2010 and 2018

search for the QCD critical point and signatures of a phase transition between hadronic gas and QGP phases. In order to increase detector acceptance and particle identification capabilities, three major subsystem upgrades were proposed must be ready till the end of 2018: Event Plane Detector (EPD), the inner TPC upgrade (iTPC) and the endcap Time-Of-Flight detector (eTOF). The EPD was fully installed and became operational for the run year 2018, replacing the Beam-Beam Counter (BBC) as a minimum-bias trigger detector. EPD allows one to carry out forward measurements of both centrality and event plane determination reducing systematic uncertainty due to autocorrelations for midrapidity analysis. This new detector consists of two disks that are placed on both sides of the STAR detector. EPD has pseudorapidity acceptance of $2.1<|\eta|<5.1$ with 16 radial segments and 24 azimuthal segments.

The iTPC will increase the acceptance of the TPC up to $|\eta|=1.5$ increasing number of readout pad rows from 13 to 40 and maximum number of hits per track from 45 to 72 . It will 
improve the $d E / d x$ resolution, and allow tracks to be reconstructed down to $p_{T}$ of $60 \mathrm{MeV} / \mathrm{c}$. A single inner sector was installed for tests for the run year 2018 and the full complement of 24 will be installed for the run year 2019.

The eTOF will be installed on one side of STAR, which will extend PID capabilities at forward rapidity. Three modules of eTOF were installed behind one of the TPC sectors for the run year 2018 and the full complement of 36 will be installed for the run year 2019. Combining all three detector systems will reduce significantly the systematic uncertainties of many observables and improve the statistical precision of STAR BES measurements.

\section{Summary}

STAR has presented a variety of experimental results in a wide energy $\sqrt{s_{N N}}$ range from $4.5 \mathrm{GeV}$ to $200 \mathrm{GeV}$ obtained in the fixed-target and collider modes. A systematic study of collision energy dependence of strange particles indicates the constituent quark coalescence. Results on heavy flavor production measured with HFT include the directed and elliptic flows of $D^{0}$ mesons and reconstruction of $\Lambda_{c}$ baryons, which supports the idea of charm thermalization at RHIC energies. Global $\Lambda$ polarization measurements in $\mathrm{Au}+\mathrm{Au}$ collisions previously made for the energies of the BES now has been presented revealing a non-zero effect for the highest RHIC energy $200 \mathrm{GeV}$. These observations can provide a new insight on the vorticity in heavy-ion collisions. Three detector upgrades are expected to be ready prior to the run year 2019 for the upcoming Beam Energy Scan phase II. The iTPC, eTOF and EPD detectors will improve the acceptance, particle identification capabilities, and reduce systematic uncertainties for many observables.

\section{References}

[1] S. Voloshin and Y. Zhang, Z. Phys. C 70, 665 (1996)

[2] L. Adamczyk et al. [STAR Collab.], Phys. Rev. Lett. 120, 062301 (2018)

[3] L. Adamczyk et al. [STAR Collab.], Phys. Rev. Lett. 112, 162301 (2014)

[4] J. C. Dunlop, M. A. Lisa and P. Sorensen, Phys. Rev. C 84, 044914 (2011)

[5] H. Qiu [STAR Collab.], Nucl. Phys. A 931, 1141 (2014)

[6] L. Adamczyk et al. [STAR Collab.], Phys. Rev. Lett. 118, 212301 (2017)

[7] B. B. Abelev et al. [ALICE Collab.], Phys. Lett. B 734, 314 (2014)

[8] L. Adamczyk et al. [STAR Collab.], Nature 548, 62 (2017)

[9] J. Adam et al. [STAR Collab.], Phys. Rev. C 98, 014910 (2018)

[10] I. Karpenko and F. Becattini, Eur. Phys. J. C 77, 213 (2017)

[11] H. Li, L. G. Pang, Q. Wang and X. L. Xia, Phys. Rev. C 96, 054908 (2017)

[12] O. Rogachevsky, A. Sorin and O. Teryaev, Phys. Rev. C 82, 054910 (2010)

[13] L. Adamczyk et al. [STAR Collab.], Phys. Rev. Lett. 112, 162301 (2014)

[14] H. Liu et al. [E895 Collab.], Phys. Rev. Lett. 84, 5488 (2000)

[15] L. Adamczyk et al. [STAR Collab.], Phys. Rev. C 97, 054909 (2018) 